\title{
ULTRASONIC IMPEDOMETRIC STUDIES IN A SMECTIC B LIQUID CRYSTAL (BBOA) $\left({ }^{*}\right)$
}

\author{
Y. THIRIET and P. MARTINOTY \\ Laboratoire d'Acoustique Moléculaire (**), Université Louis-Pasteur \\ 4, rue Blaise-Pascal, 67070 Strasbourg Cedex, France
}

(Reçu le 10 janvier 1975, accepté le 27 février 1975)

\begin{abstract}
Résumé. - En utilisant la technique de réflexion d'une onde ultrasonore de cisaillement sur un interface solide-liquide, nous avons étudié le comportement d'un smectique B soumis à une contrainte de cisaillement dans le cas où le vecteur d'onde est parallèle à la normale aux couches. Nous n'avons pas observé de transmission de l'onde ultrasonore. En outre, nous avons mis en évidence un processus de relaxation qui apparaît, à la fois, dans les phases $\mathrm{A}$ et $\mathrm{B}$, dans le domaine des fréquences étudiées, et qui conduit à un module de cisaillement apparent, différent de zéro. Nous discutons nos résultats dans le cadre de la théorie de de Gennes-Sarma.
\end{abstract}

\begin{abstract}
The behaviour of a smectic B phase (BBOA) has been investigated by means of shear wave reflectance techniques in the case of propagation parallel to the normal of the layers. No transmission of the shear wave has been observed. In addition, the experimental results in the A and $B$ phases show a relaxational process, which leads to an apparent finite value for the shear modulus. Our results are discussed in the frame-work of the de Gennes-Sarma model and appear to support this model.
\end{abstract}

The smectic phases are layered systems. In contrast to the $\mathrm{A}$ and $\mathrm{C}$ phases, which are two dimensional liquids, the $B$ phase shows an ordering inside the layers $[1,3]$. The difference between the $B$ phase and a solid is not yet clearly defined. It has been suggested that the B phase may correspond to a plastic crystal [2] or to a system of weakly coupled solid layers [4, 5].

To understand the structure of the B phase many experiments have been performed using X-ray scattering $[1,3]$, quasi elastic neutron scattering [6], Brillouin scattering [7], and E.P.R. measurements [8].

In this letter, we present the first measurements of ultrasonic shear waves propagating along the normal of the layers.

We only sketch the most important experimental features since a more complete description will be given elsewhere. We have studied samples of 40.8 , usually called BBOA, |-N-(p-butyloxybenzylidenep.n octylaniline | [9]. The samples are placed between two trimethylammonium-bromid treated fused silica rods without spacers in such a manner that the applied layer should be as thin as possible, and heated to the isotropic phase (see Fig. 1). Upon slow cooling, we successively obtain a homeotropic monodomain of

(*) Work supported by Délégation Générale à la Recherche Scientifique et Technique under contract no 7470458.

$(* *)$ Associated with C.N.R.S. nematic, smectic $A$ and $B$ phases. If no disturbance occurs, the B phase supercools. Microscopic observations between crossed polarizers confirm the orientation of the samples. Calorimetry experiments are used to determine the transition temperatures. The phase diagram is the following :

$$
\mathrm{C} \rightarrow 37.7 \mathrm{~S}_{\mathrm{B}} \rightarrow 49.2 \mathrm{~S}_{\mathrm{A}} \rightarrow 63.7 \mathrm{~N} \rightarrow 78.6 \mathrm{I} \text {. }
$$

The shear mechanical properties of the specimen are determined by a reflection technique, briefly, by generating pulsed ultrasonic shear waves in a solid rod and reflecting them at the interface between the rod and the test liquid (see Fig. 1). A train of echoes, which can be displayed on an oscilloscope,

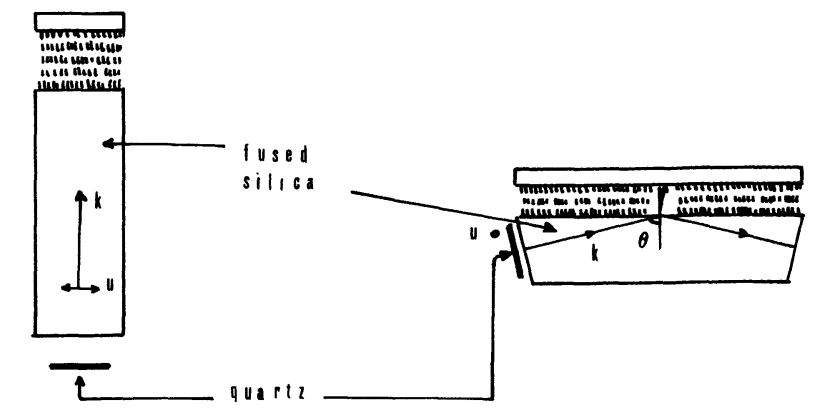

FIg. 1. - Acoustic system : Normal and oblique incidence. $k$ is the wave vector; the vibration $u$ is parallel in both cases to the reflecting surface. 
is set up in the rod. The falloff, in decibels, and the phase shift per echo is measured for both the quartzair and quartz-liquid interface. The complex reflection coefficient at the interface at normal incidence is given by the formula :

$$
r \mathrm{e}^{-i \varphi}=\frac{Z_{\mathrm{s}}-Z_{1}}{Z_{\mathrm{s}}+Z_{1}}
$$

where $Z_{\mathrm{s}}$ is the characteristic impedance of the silica $\left(Z_{\mathrm{s}}=8.29 \times 10^{5}\right.$ dyne $\left.\mathrm{s} \mathrm{cm}^{-3}\right)$ and $Z_{1}=R+i X$ the characteristic impedance of the liquid. This impedance is defined as the ratio of shear stress to transverse particle velocity.

In terms of $r$ and $\varphi$ the values of $R$ and $X$ are given by :

$$
R=Z_{\mathrm{s}} \frac{1-r^{2}}{1+r^{2}+2 r \cos \varphi} \sim Z_{\mathrm{s}} \frac{1-r}{1+r}
$$

for small values of $\varphi$

$$
X=Z_{\mathrm{s}} \frac{2 r \sin \varphi}{1+r^{2}+2 r \cos \varphi} \sim Z_{\mathrm{s}} \frac{2 r \varphi}{(1+r)^{2}} .
$$

The expressions for the complex dynamic stiffness $G=G^{\prime}+i G^{\prime \prime}$ and dynamic viscosity $\eta=\eta^{\prime}-i \eta^{\prime \prime}$ are the followings :

$$
\begin{aligned}
G^{\prime} & =\frac{R^{2}-X^{2}}{\rho} & \eta^{\prime} & =\frac{2 R \dot{X}}{\rho \omega} \\
G^{\prime \prime} & =\frac{2 R X}{\rho} & \eta^{\prime \prime} & =\frac{R^{2}-X^{2}}{\rho \omega} .
\end{aligned}
$$

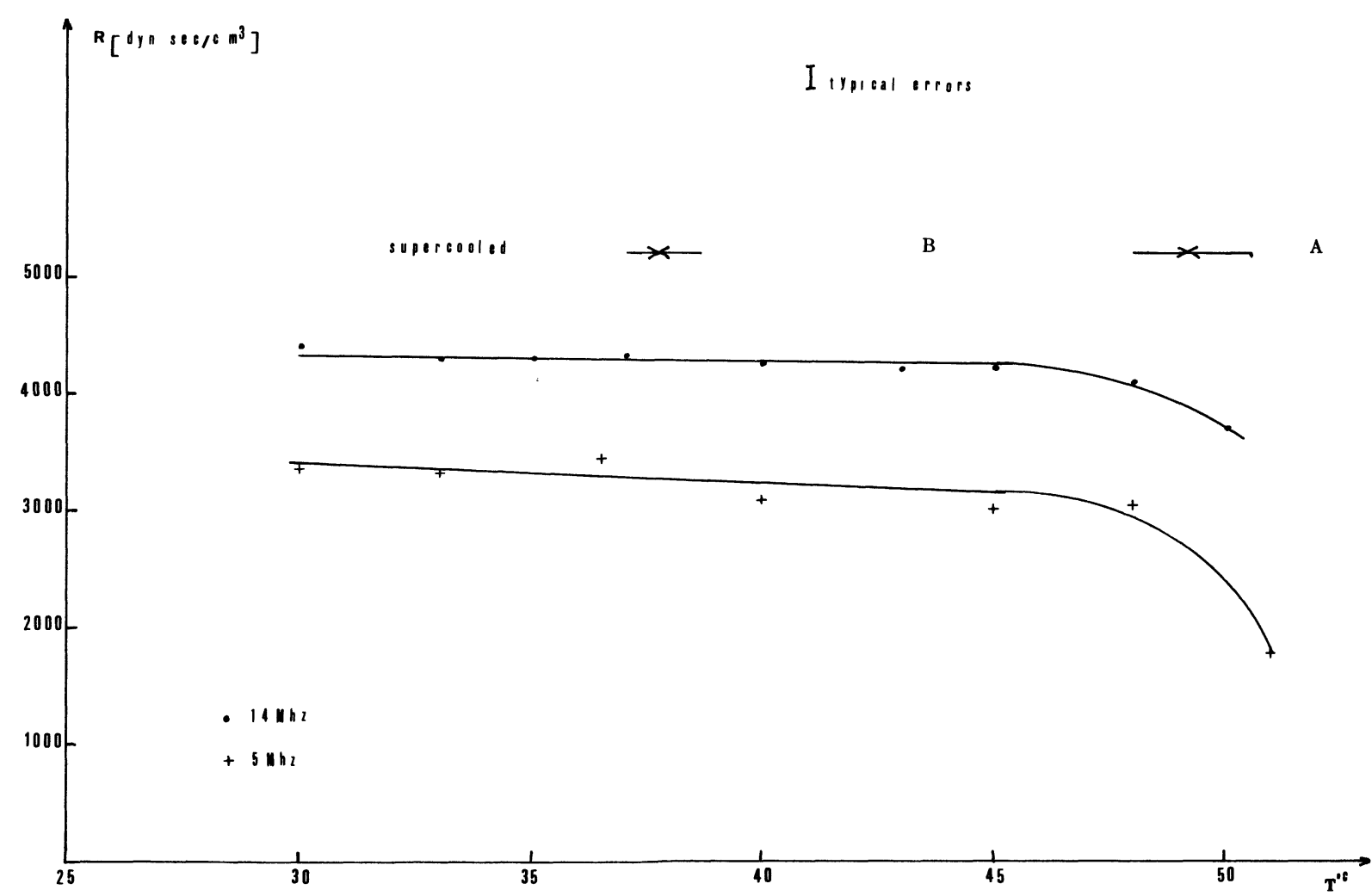

By reflecting the shear wave from the interface at oblique incidence, the sensitivity of the method is increased because, in the above expressions, $Z_{\mathrm{s}}$ is now $Z_{\mathrm{s}} \cos \theta$, where $\theta$ is the angle of incidence from the normal.

We can expect two different behaviours from our experiment.

If we consider the smectic $B$ phase as a plastic crystal, the velocity of propagation in the sample remains finite, and only the rotational disorder contributes to the damping. At the interface, the wave is transmitted through the specimen and partially reflected. Thus, one might observe a pattern very similar to the one shown in photograph A.

On the other hand, when the shear modulus $c_{44}$ vanishes, the acoustical properties are completely different. The layers can slip freely on top of each other while still remaining in a two-dimensional ordered structure. In other words, we have a set of lubricated planes where the aliphatic chains is the lubricant. In this case, most of the wave is reflected; this behaviour has been observed as shown in photograph $B$. We have obtained the same pattern at normal and oblique incidence.

We have measured the real part $R$ of the shear impedance both at normal and oblique incidence. The results are similar within the experimental error $(10 \%)$. This suggests that the shear impedance of the $\mathrm{B}$ phase is very low compared to $Z_{\mathrm{s}}$. Thus, the transmitted wave for the oblique incidence is nearly parallel to the normal of the layers. We present, in

$$
\text { I typical errors }
$$

FIG. 2. - Temperature dependence of the real part $R$ of the shear mechanical impedance at $5 \mathrm{MHz}$ and $14 \mathrm{MHz}$

- Typical echo pattern, corresponding to the reflected wave, observed at $5 \mathrm{MHz}$. 

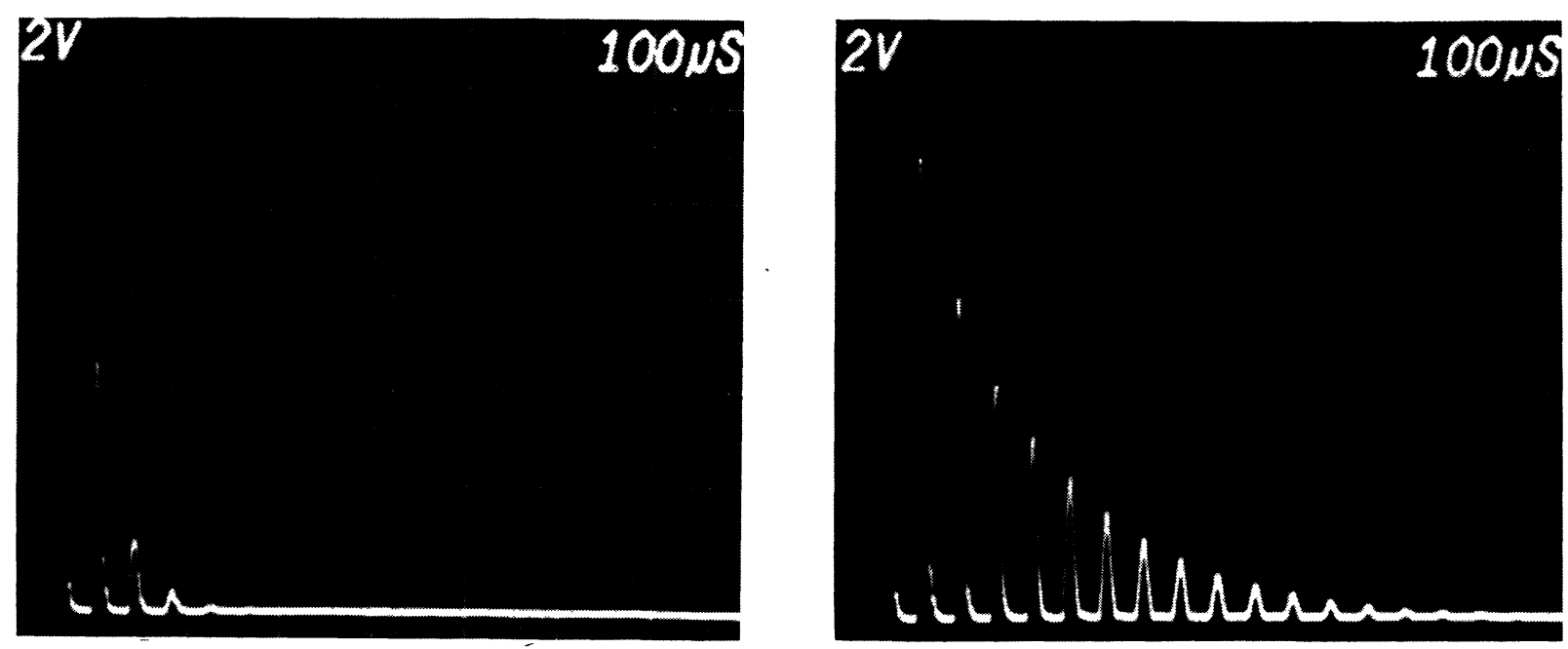

a) in the crystallized phase at $30^{\circ} \mathrm{C}$ (photograph $\mathrm{A}$ ) ;

b) at the same temperature when the smectic B is supercooled (photograph B). A similar pattern is obtained when the bar is loaded with a smectic A.

The same excitation pulse has been applied in the two cases a and b. The sweep rate is $100 \mu / \mathrm{div}$ and the gain $2 \mathrm{~V} / \mathrm{div}$.

figure 2, the experimental values of $R$ at $5 \mathrm{MHz}$ and $14 \mathrm{MHz}$ obtained from oblique incidence. $R$ is of the same order of magnitude as in a conventional liquid or a smectic A phase [10].

We have also performed preliminary measurements on the imaginary part $X$ of the shear impedance. We have observed that $R \neq X$. These results are, for the moment, not very accurate $\left({ }^{1}\right)$, but significant. This suggests the existence of a relaxational process (for a non relaxing liquid $R=X$ ). Another test is given by the ratio $2 R^{2} / \rho \omega$ which is not constant.

The first implication is that the effective viscosity coefficient is frequency dependent. Furthermore, this frequency dependence is not specific for the $B$ phase. We also observed this effect in the $A$ phase of $\mathrm{BBOA}$ and on another compound [10],

$$
\left(\mathrm{C}_{7} \mathrm{H}_{15}-\langle\varnothing\rangle-\underset{\mathrm{O}}{\mathrm{N}}=\mathrm{N}\langle\varnothing\rangle-\mathrm{C}_{7} \mathrm{H}_{15}\right)
$$

which has only a smectic A phase.

One possible explanation is that the conformational changes in the long aliphatic chains submitted to the streess are at the origin of this phenomenon.

The second implication is that the compound can be considered as a liquid of stiffness $G^{\prime}$ (i.e. $c_{44}$ ) given by formula (3). Thus, relaxation effects lead to an apparent finite value of $c_{44}$.

Let us discuss our results in conjunction with other recent experimental data.

- One experiment, by X-ray diffraction [3] on the same compound, shows a very short correlation length perpendicular to the layers (about two inter-

( $\left.{ }^{1}\right)$ Due to the very small phase change $\varphi$ caused by application of the smectic. This phase change, typically $1^{\circ}$, corresponds to a change of about $0.30 \mathrm{~Hz}$ in the pulse superposition method [11]. layers). (Compounds such as TBBA (4 n Terephtal bis butyl aniline) with large correlation lengths are described as a plastic crystal).

- A study by E.P.R. on another compound with very long aliphatic chains suggests that the solid $\rightleftarrows$ smectic B transition corresponds to a melting of the aliphatic chains.

- Our ultrasonic study confirms these two experiments because we have observed no transmission of the shear wave and a real part of the shear impedance is of the same order of magnitude as for a conventional liquid.

All these results tend to support the de GennesSarma model of the $B$ phase, now the question is to know if $c_{44}=0$ for $\omega=0$.

From our experiment we cannot calculate $c_{44}$ (because the accuracy on $X$ is, for the moment, too poor), but the upper limit given by $R^{2} / \rho$. We observe a decrease of $R^{2}$ (i.e. $c_{44}$ ) when the frequency goes from $14 \mathrm{MHz}$ to $5 \mathrm{MHz}$. An experiment by Brillouin scattering [7] on $\beta$-methyl butyl p | (p-methoxybenzylidene) amino / cinnamate indicates that $c_{44}$ does not vanish, although it is much smaller than the other moduli. We have seen previously that relaxation effects lead to an apparent finite value of $c_{44}$. Consequently, this apparent finite value of $c_{44}$ could result from a relaxation effect similar to the one we have observed. This behaviour at high frequency (Brillouin and ultrasonic) does not exclude the possibility of $c_{44} \rightarrow 0$ for $\omega \rightarrow 0$, but the fact that the relaxation effect appears also in the A phase and that the real part of the shear impedance is of the same order of magnitude in the $\mathrm{A}$ and $\mathrm{B}$ phases tends to favour $c_{44} \rightarrow 0$ for $\omega \rightarrow 0$.

On the other hand, the origin of the relaxational process is not well understood, but is presumably related to the change of conformations of the aliphatic chains. Accurate measurements of $X$ and experi- 
ments at other frequencies in the $B$ and $A$ phases (in order to get values of the effective viscosity and stiffness coefficients and the contribution of the relaxational process) are in progress and will clarify this point.
Acknowledgments. - We thank Dr. S. Candau for very stimulating discussions, Dr. Mioskowski for the supply of BBOA and Miss Gonthier for the calorimetry experiments.

\section{References}

[1] Levelut, A. M., Lambert, M., C. R. Hebd. Séan. Acad. Sci. B 272 (1971) 1018.

[2] Doucet, J., Levelut, A. M., Lambert, M., Mol. Cryst., to be published.

[3] Levelut, A. M., Doucet, J., Lambert, M., J. Physique 35 (1974) 773.

[4] De Gennes, P. G., Sarma, G., Phys. Lett. 38A (1972) 219.

[5] De Gennes, P. G., Mol. Cryst. Liq. Cryst. 21 (1973) 49.

[6] Hervet, H., Volino, F., Dianoux, A. J., Lechner, R. E., J. Physique Lett. 35 (1974) L-151.
[7] Liao, Y., Clark, N. A., Pershan, P. S., Phys. Rev. Lett. 30 (1973) 639.

[8] Dvolaitsky, M., Poldy, F., Taupin, C., Phys. Lett. 45A (1973) 454.

[9] 40.8 was synthetized by Dr. Mıskowskı in Strasbourg.

[10] Kiry, F., MarTinoty, P., to be published.

[11] For the pulse superposition method see for instance : MC Skimin, H. J., Andreath, P., J. Acoust. Soc. Am. 34 (1962) 609.

For the impedometer technique see for instance : BARLOW, A. J., Lamb. J.. Proc. R. Soc. A 253 (1969) 52. 